The authors call for new treatment strategies directed at reducing the severity of stroke, as these might reduce the incidence of post-stroke epilepsy.

Marie Lofthouse

Original article Lossius MI et al. (2005) Poststroke epilepsy: occurrence and predictors-a long-term prospective controlled study (Akershus Stroke Study). Epilepsia 46: 1246-1251

\section{Clinical profiling of spinocerebellar ataxia genetic subtypes}

Spinocerebellar ataxia (SCA) has several genetically defined subtypes. A recent multicenter study investigated the clinical symptoms associated with these subtypes, to identify patterns of clinical features that might distinguish between the types of SCA.

Maschke and colleagues recruited 127 patients with SCA types 1-8, and carried out a neurological assessment of their symptoms using a worksheet comprising 33 symptoms and signs. They then calculated the probability of each trait being present in a particular SCA type, and used this to rate the predictive value of individual clinical features for each type of SCA. These were combined to form a Bayesian classifier, which was later simplified to form an algorithm that separated SCA types into three groupsthose with upper motor neuron involvement, those with neuropathy, and those with a predominantly cerebellar syndrome.

Overall, the Bayesian classifier was able to correctly predict the type of SCA in $78 \%$ of patients. The most accurate predictions were achieved for SCA7 (sensitivity 100\%, specificity 98\%), SCA8 (sensitivity $91 \%$, specificity $97 \%$ ), and SCA5 (sensitivity $88 \%$, specificity $95 \%$ ).

Concluding that many subtypes of SCA do exhibit characteristic clinical features, the authors note that, for practical reasons, an algorithm might be more easily adopted by medical centers than a Bayesian classifier. In addition, they state that algorithms based on detailed clinical data could help prioritize genetic testing for specific types of SCA, thus helping to reduce the costs of such tests.

Christine Kyme

Original article Maschke $\mathrm{M}$ et al. (2005) Clinical feature profile of spinocerebellar ataxia type 1-8 predicts genetically defined subtypes. Mov Disord [doi: 10.1002/mds.20533]

\section{Spinal cord compression due to metastasis: surgical resection is superior to radiotherapy}

A randomized multicenter trial has demonstrated that surgical resection is more effective than radiotherapy in patients undergoing surgery for spinal cord compression caused by metastatic cancer. Although standard treatment is radiotherapy and steroids, data from uncontrolled surgical studies encouraged Patchell et al. to investigate the effectiveness of surgical treatment, using ambulation as a primary endpoint.

The original intention to randomize 100 patients into two treatment groups-one receiving surgery within $24 \mathrm{~h}$ of randomization followed by radiotherapy, the other beginning radiotherapy alone within $24 \mathrm{~h}$. Both groups received steroids. Recruitment was halted, however, after interim analysis showed surgical treatment to be superior in terms of ambulation rates ( $84 \%$ vs $57 \%, P=0.001)$, duration of ambulatory ability, recovery of ambulatory ability, and use of corticosteroids and analgesics.

Eligible patients fulfilled strict criteria, including tissue-proven cancer diagnosis (not radiosensitive cancer, or cancer of CNS or spinal column origin), a single point of MRI-confirmed spinal-cord displacement, no unrelated neurological problems, and no previous spinal radiation preventing delivery of the study radiation dose. Also, patients could not have had total paraplegia for $>48 \mathrm{~h}$ prior to study entry. Although the authors concede that their conclusions cannot be extrapolated to patients not fulfilling these criteria, the study shows surgical treatment to be markedly superior to radiotherapy in this context.

Rebecca Doherty

Original article Patchell RA et al. (2005) Direct

decompressive surgical resection in the treatment of spinal cord compression caused by metastatic cancer: a randomised trial. Lancet 366: 643-648

\section{Increased lesion load and atrophy of white and gray matter in multiple sclerosis patients}

The relationship between the level and location of brain atrophy and the clinical manifestations of multiple sclerosis (MS) remains to be clarified. Variations in MRI techniques and failure 